\title{
A Semi-Empirical Noise Modeling Method for Helicopter Maneuvering Flight Operations
}

\author{
Eric Greenwood* \\ NASA Langley Research Center
}

\author{
Fredric H. Schmitz ${ }^{\dagger}$ Richard D. Sickenberger ${ }^{\ddagger}$ \\ University of Maryland
}

A new model for Blade-Vortex Interaction noise generation during maneuvering flight is developed in this paper. Acoustic and performance data from both flight and wind tunnels are used to derive a non-dimensional and analytical performance/acoustic model that describes BVI noise in steady flight. The model is extended to transient maneuvering flight (pure pitch and roll transients) by using quasisteady assumptions throughout the prescribed maneuvers. Ground noise measurements, taken during maneuvering flight of a Bell 206B helicopter, show that many of the noise radiation details are captured. The result is a computationally efficient Blade-Vortex Interaction noise model with sufficient accuracy to account for transient maneuvering flight. The code can be run in real time to predict transient maneuver noise and is suitable for use in an acoustic mission-planning tool.

\section{Notation}

A

$a_{0}$

$B$

b

$C$

$C_{T}$

$C_{p^{\prime}}$

$C_{p}$

$C_{v}$

$D$

$M$

$\hat{M}$

$M_{H}$

$M_{r}$

$\hat{n}$

$P$

$p_{L}^{\prime}$

$r$

$\hat{r}$

$\bar{r}$

$\bar{r}_{0}$

$\bar{r}_{v}$

$R$

$S$

$S$

$\bar{S}$

$t$

Mean Inflow Coefficient

Ambient Speed of Sound

Number of Rotor Blades

Thrust Coefficient

Section Mach Number

Mach Direction Vector

Hover Tip Mach Number

Surface Pressure

Propagation Distance

Rotor Radius

Blade Surface Area

Reduced Time

Time of Observation
Longitudinal Inflow Coefficient

Lateral Inflow Coefficient

Acoustic Pressure Coefficient

Blade Surface Pressure Coefficient

Vortex Core Growth Coefficient

Wake Contraction Coefficient

Mach Number along Propagation Direction

Surface Normal Direction

Acoustic Perturbation Pressure due to Loading

Propagation Direction Vector

Non-Dimensional Propagation Distance

Non-Dimensional Vortex Core Radius

Non-Dimensional Vortex Radius

Non-Dimensional Blade Surface Area $\bar{t} \quad$ Non-Dimensional Time of Observation

$v_{\theta} / \Omega R$ Non-Dimensional Swirl Velocity

$\vec{x} \quad$ Non-Dimensional Cartesian Coordinate Vector

$X \quad$ Indicial Function

$Y \quad$ Indicial Function

$\hat{i}, \hat{j}, \hat{k} \quad$ Tip-Path-Plane Unit Direction Vectors

$x, y, z \quad$ Non-Dimensional Tip-Path-Plane Coordinates

$\alpha \quad$ Blade Element Angle of Attack

$\bar{\gamma}_{v} \quad$ Non-Dimensional Vortex Filament Strength

$\gamma_{0} \quad$ Mean Vortex Strength Coefficient

$\gamma_{1 c} \quad$ Longitudinal Vortex Strength Coefficient

$\gamma_{1 s} \quad$ Lateral Vortex Strength Coefficient

$\Gamma_{0} \quad$ Hover Nominal Tip Vortex Circulation Strength

$\bar{\Gamma} \quad$ Non-Dimensional Circulation

$\lambda_{i} \quad$ Local Induced Inflow Ratio

$\lambda_{0} \quad$ Mean Inflow Ratio

$\lambda_{1} \quad$ Wake Contraction Parameter

$\lambda_{1 c} \quad$ Slope of Longitudinal Inflow

$\lambda_{1 s} \quad$ Slope of Lateral Inflow

$\mu \quad$ Advance Ratio

$\rho_{0} \quad$ Ambient Density

$\tau \quad$ Time of Emission

$\bar{\tau} \quad$ Non-Dimensional Time of Emission

$\chi \quad$ Wake Skew Ratio

$\psi_{b} \quad$ Rotor Blade Azimuth

$\psi_{v} \quad$ Vortex Filament Azimuth

$\phi \quad$ Wake Age

$\Omega \quad$ Rotor Rotational Speed

() Derivative with Respect to $\psi$

${ }^{*}$ Research Aerospace Engineer, eric.greenwood@nasa.gov

†Senior Research Professor, fschmitz@eng.umd.edu

† Graduate Research Assistant, rdsicken@umd.edu

Presented at the American Helicopter Society 68th Annual Forum, Fort Worth, TX, May 1-3, 2012. This is a work of the U.S. Government and is not subject to copyright protection in the U.S. 


\section{Background}

Acoustic prediction is becoming an increasingly important component of helicopter mission planning for both civil and military applications. Estimates of helicopter noise radiation to observers on the ground are required to design low noise helicopter operations that minimize community annoyance or the acoustic detection of helicopters. To be useful, these estimates should be available in near real-time and be reasonably accurate.

Several empirical noise-mapping methods are currently used for mission planning. (Refs. 1-6) These models assume that any mission profile can be represented as a sequence of steady-flight segments. Each segment is associated with a description of the noise radiated by the helicopter during that steady-state flight condition. An estimate of the noise generated during the mission is obtained by projecting the acoustic data from each flight segment to the ground. The relation between noise radiation and steady-state operating condition is derived for a particular helicopter from a set of ground-noise measurements. This data is gathered by flying the helicopter in a steady-state flight condition over an array of microphones. This process is typically repeated for a range of airspeeds and flight-path angles. The measured acoustic data for each flight condition is stored and may be used to characterize the acoustic radiation of the helicopter.

These noise-mapping methods work quite well for describing the noise radiation during steady-flight. In particular, main rotor Blade-Vortex Interaction (BVI) noise, which is the dominant source of noise for many helicopters during approach, has been shown to be a strong function of the mean inflow through the rotor disk, which is a strong function of both the airspeed and flight-path angle of the helicopter. As the helicopter descends, the shed tip vortices of the main rotor are positioned close to the rotor's tip-pathplane, causing strong BVI noise.

A method of extending these empirical methods to predict the noise radiation for a helicopter executing slow-tomoderate maneuvers has also been developed. (Ref.7) Constant acceleration/deceleration quasi-steady segments are inserted in between the steady-state segments to approximate the conditions of slow-moderate maneuvering flight. The radiated noise is calculated by determining an equivalent steady condition in the measured acoustic data set and then interpolating that data to find the noise radiation in the direction of the observer. This Quasi-Static Acoustic Mapping (Q-SAM) method has been validated in several experiments and has been incorporated in empirical noisemapping codes. (Refs. 8,9)

In practice, helicopters often perform more aggressive transient maneuvers during typical flight operations, such as when approaching landing areas or when flying nap-ofthe-earth to evade detection. The external noise of the helicopter rapidly changes during these transient flight con- ditions. Similar to steady descending flight, one of the most prominent changes occurs when a transient maneuver causes the rotor wake to pass in close proximity to the tip-path-plane resulting in increased BVI noise radiation. Much of this BVI noise is directed towards the ground in the direction of forward flight. However, since the rotor tippath-plane may move significantly out of the plane of the horizon during a maneuver, BVI noise radiation can also be directed towards the horizon. (Ref. 10) It may not be possible to model the increase in BVI noise radiation due to transient maneuvering flight to the accuracy desired using the quasi-static (Q-SAM) method alone. The dynamic movement of the rotor and the movement of the shed vortices during the transient maneuver are likely to be significant, and may require more accurate physics-based modeling.

First principles modeling of BVI noise has been attempted for the past forty years. In general, it has been shown that if the wake details are accurately captured, the noise can be predicted quite well. (Ref. 11) However, the information about the wake at the required level of detail is not normally available during the flight test of full helicopters. In addition, the large computational time of first principles modeling methods precludes their use for near real-time noise prediction.

Recent results from pure first principles theoretical modeling of helicopter BVI noise during transient maneuvering flight has suggested that very high noise levels might be achieved during aggressive maneuvers. These results were produced using a free-wake model that captured the bundling of trailed vortices under certain maneuvers that, when coupled with rotor aerodynamic and noise prediction codes, led to very high predicted noise levels. This phenomenon was called "super BVI," and was associated with vortex bundling; producing BVISPL levels that were predicted to be stronger than the effect of individual vortices that are normally encountered during descending flight. (Refs. 12, 13) However, the results for these very aggressive maneuver predictions have not been validated against experimental wake or acoustic measurements.

Analysis of acoustic data collected during recent measurements of a Bell 206B helicopter during maneuvering flight has indicated that, in some conditions, BVI noise radiated during a maneuver initiated from level flight could set the aural detection distance of rotorcraft. (Ref. 10) Several moderately aggressive transient maneuvers were flown that are typically encountered in operational situations. While BVI noise levels on the Bell 206B helicopter were significant, no evidence of "super BVI" significantly stronger than that achieved during steady-state descending flight was observed over the range of maneuvers flown during the program. Using a rigid-wake model, and keeping track of discrete BVI events, the BVI noise observed during maneuvering flight was found to correspond to the same types of interactions that occur during steady-state descending flight. 


\section{Objective}

The objective of this paper is to develop an accurate and computationally efficient analytical model of helicopter Blade-Vortex Interaction (BVI) noise during transient maneuvering flight. The model is based on a nondimensional and semi-empirical approach, called Fundamental Rotorcraft Acoustic Modeling from Experiments (FRAME). (Ref. 14) FRAME employs parameter identification techniques to adapt analytical models of the rotor noise sources to fit measured acoustic data captured over a wide range of steady-state operating conditions. Transient maneuvers are treated as dynamic deviations from these steady-state conditions. Using this approach, a semiempirical model for a specific helicopter is produced which can be used to predict the main rotor BVI noise radiation of a maneuvering helicopter to the accuracy required for mission planning for helicopter operations.

\section{Experimental Data Sets}

Measured data from several flight and wind tunnel test programs have been used in the development of the transient maneuver flight modeling presented in this paper. One acoustic data set was gathered some years ago in the German-Dutch Windtunnel (DNW) anechoic wind tunnel on the $1 / 7$ th scale Operational Loads Survey (OLS) model rotor. (Ref. 15) The acoustic data set spanned a large range of rotor operating conditions and focused on the key parameters that cause Blade-Vortex-Interaction (BVI) noise. Microphones were placed in the free-stream of the open jet at three rotor radii from the rotor hub directly ahead of the helicopter, and thirty degrees to either side and in the plane of the rotor. Microphones were also positioned at two additional elevation angles below the rotor at thirty and forty-five degrees, as shown in Figure 1 The model rotor acoustic data were of high quality and, when appropriately scaled, were shown to match full-scale acoustic data taken on a similar rotor taken in flight using a flying microphone. (Ref. 16) The microphone was located on a quiet fixed wing aircraft that was flown in formation at a fixed distance from the helicopter

Acoustic data were also gathered on a full-scale Bell 206B helicopter during two separate flight test programs. The first test program was funded by the Center for Rotorcraft Innovation (CRI) and conducted by University of Maryland, NASA, and the Army at the NASA Ames Research Center at Moffett Field, CA, in 2006. (Ref. 8) This test was unique in that it featured an array of microphones attached to a modified crop-dusting spray rig that enabled noise measurements to be made in a frame moving with the helicopter, shown in Figure 2 Noise measurements were simultaneously made on the ground and were used to identify BVI noise radiation patterns during steady and quasi-steady flight. It should be noted that the addition of the measurement rig had a substantial impact on the drag of the heli-

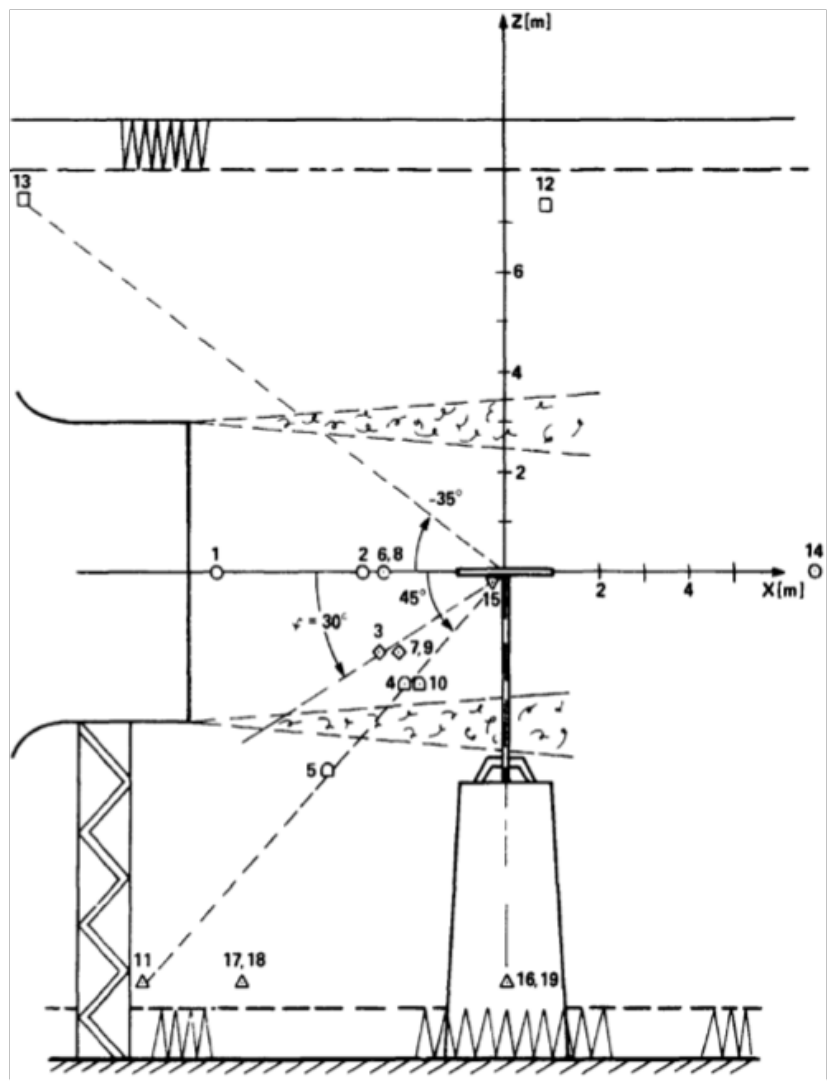

Fig. 1. A side-view of the $1 / 7$ th Scale OLS test microphone layout.

copter, increasing the forward tilt of the rotor during both level and descending flight, and consequently requiring a steeper descent angle to achieve maximum BVI noise radiation.

A second Bell 206B helicopter flight test program was conducted by a Bell Helicopter, NASA, Army, and University of Maryland test team on a rented farm in Hollister, California in June 2007. The helicopter was flown in a "clean" configuration, without the inflight microphone array, as shown in Figure 3. The test site was selected for its low background noise levels and very low ambient winds. In addition to steady-state noise and performance measurements, constant acceleration/deceleration maneuvers and steady-state turning flight maneuvers were flown to confirm quasi-static methods of estimating noise. Transient maneuvers were flown toward the end of the program and consisted of simple cyclic pull-ups and rolls that were initiated from level steady-state flight conditions at approximately 100 feet AGL. In-flight measurements were made of aircraft performance parameters, including tip-path-plane angle of attack and inertial accelerations, in conjunction with ground-based acoustic measurements. The focus of this paper is to develop a method of estimating the radiated BVI noise that occurs during these transient maneuvers using a semi-empirical model with modeling parameters derived from steady-state flight and wind tunnel acoustics data. 


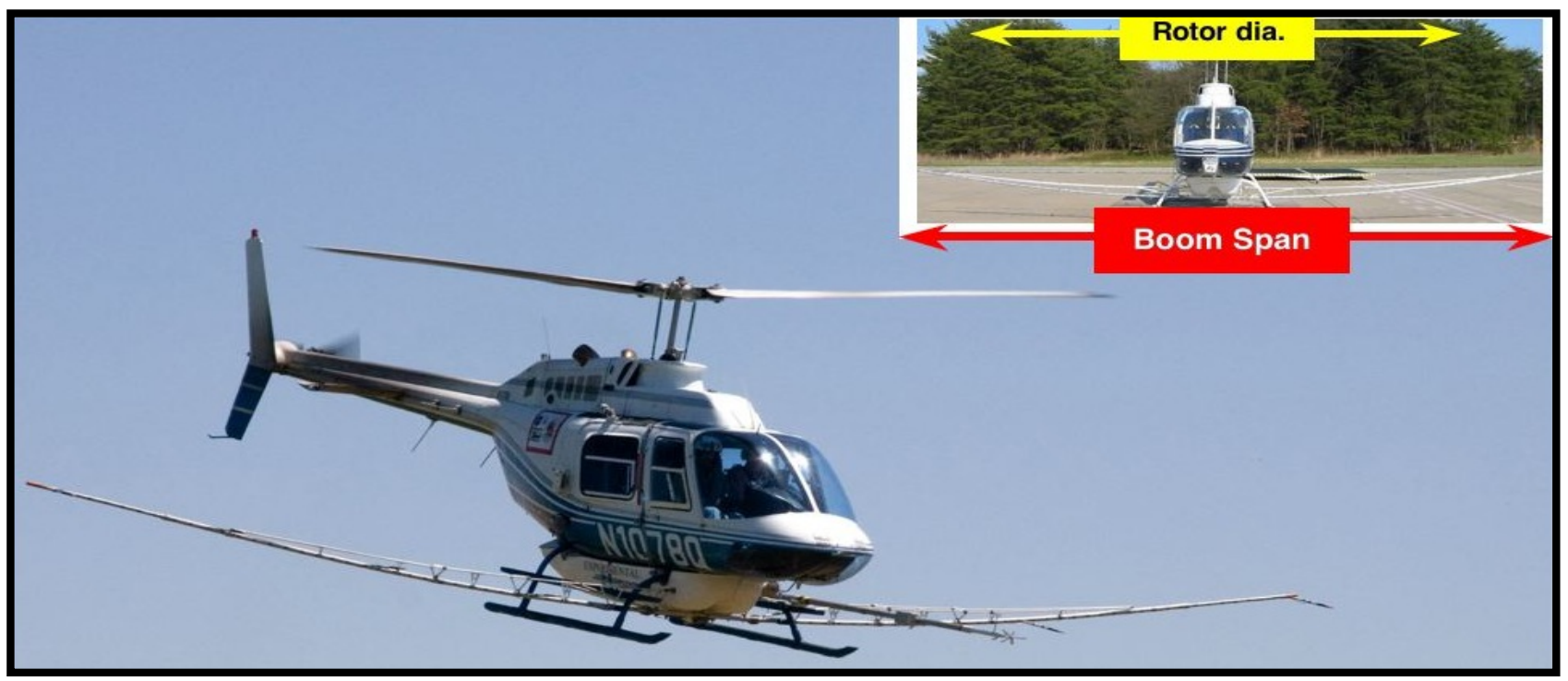

Fig. 2. The Bell 206B during the 2006 flight test program, configured with the inflight microphone array.

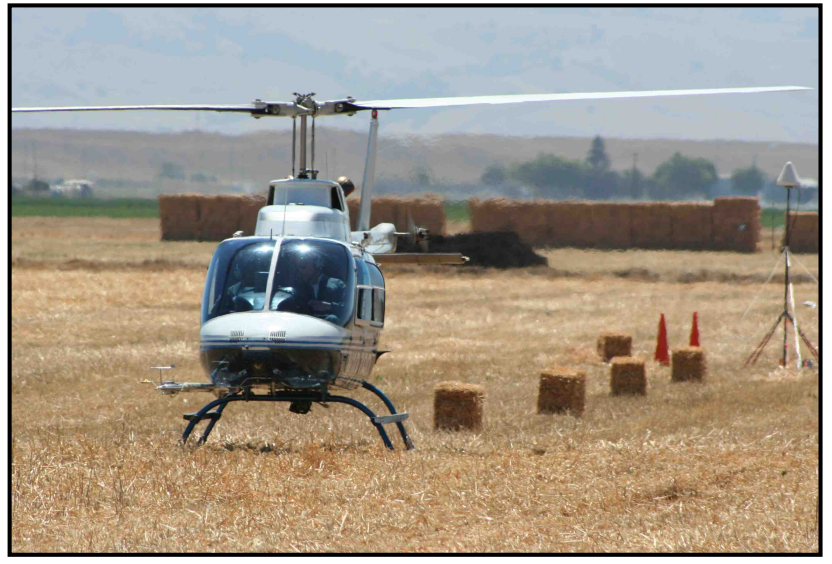

Fig. 3. The Bell 206B during the 2007 flight test program in a "clean" configuration.

\section{Steady-State FRAME Modeling}

The semi-empirical modeling method that is described in this paper is an extension of the previously developed FRAME method. (Refs. 14, 17) FRAME allows measured acoustic data, from wind tunnel experiments and flight tests of similar rotors, to be mathematically generalized to represent the noise radiation at other operating conditions. It does this by building non-dimensional phenomenological models of the major rotor noise sources that match the acoustic measurements made during flight and/or wind tunnel testing of a particular vehicle. It then uses the resulting mathematical models to predict the radiated noise that the helicopter produces at other operating conditions and radiation conditions than were previously measured.

Since BVI dominates the measured noise during transient maneuvers, a non-dimensional and phenomenological FRAME model will be developed that accurately describes
BVI in steady-state flight and may also be used to predict BVI noise during transient maneuvering flight. The focus of the paper will be on estimating the changes in the wake position relative to the tip-path-plane of the main rotor during the transient maneuver, because the "miss-distance" between the tip vortices and rotor blades changes significantly during a rapid maneuver and is a dominant factor in the generation of BVI noise.

FRAME applies a parameter identification approach to a non-dimensional assumed analytical model of the rotor noise sources in order to develop a semi-empirical model for a particular helicopter which characterizes the noise radiation as a function of the rotor operating condition. The set of governing parameters used by FRAME includes the thrust coefficient $\left(C_{T}\right)$, advance ratio $(\mu)$, hover tip Mach number $\left(M_{H}\right)$, and inflow ratio $\left(\lambda_{0}\right)$. These governing parameters define the rotor operating condition and are related through parameter identification to the dependent modeling parameters of the analytical model. This results in an analytical model that reproduces the measured noise at all known operating conditions and enables accurate estimates of noise at other operating conditions. This process is illustrated in Figure 4

A previous version of FRAME was developed to model main rotor BVI noise under steady operating conditions that used a variation on the Beddoes prescribed wake model. (Ref. 18) The Beddoes (Ref. 19) wake was modified to include FRAME dependent modeling parameters controlling the inflow distribution over the rotor disk, harmonic vortex strength distribution, tip vortex initial roll-up radius and contraction, and initial vortex core size and growth. This wake model was used in conjunction with 2D indicial aerodynamics theory (Ref. 20) to compute compact-chord BVI airloads on the rotor blades. These airloads were then used 
as an input to a linear acoustic prediction code to estimate the noise at the desired observer locations. Due to the relatively short propagation ranges of the measured data examined in this paper, the effects of atmospheric absorption have been neglected in the FRAME model.

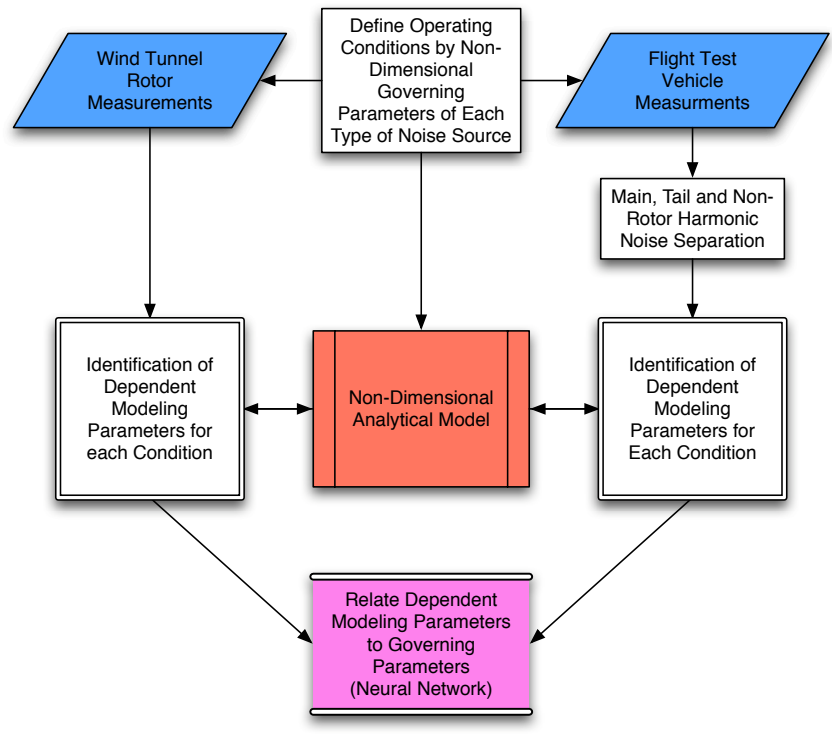

Fig. 4. A flowchart of the FRAME method for developing rotorcraft acoustic models.

\section{Steady State FRAME Noise Predictions Versus Measurement}

A FRAME modeling structure for BVI was selected and key parameters were identified using measured data for the Bell 206B helicopter in steady-state flight from the 2006 flight test program (Ref. 8) and combined with wind tunnel data from the two-bladed $1 / 7$ th scale OLS rotor in the DNW wind tunnel. (Ref. 15) The FRAME model was first used to model steady flight and compared to measured data captured from three ground-based microphones from the 2006 flight test program. Many additional comparisons between measured data and FRAME models for the Bell 206B in steady flight may be found in References 17 and 14 for BVI and other main rotor harmonic noise sources.

To recheck the ability of FRAME to reproduce steadystate BVI noise, acoustic measurements at three microphone locations were compared with FRAME predictions. Figure 5 compares the BVI noise predicted by FRAME for a steady-state descent at 60 KIAS and $-7.5^{\circ}$ flight-path angle to measured data captured at observer positions towards the advancing, center, and retreating sides of the rotor. At the time shown, the helicopter was approaching the array from a distance of 1,000 feet from the center microphone and at an altitude of 375 feet above ground level. This flight condition was known to generate strong BVI noise radiation in the Bell 206B as it was configured in the 2006 flight test. The measured data have been averaged on the main rotor blade passages in order to suppress other noise sources such as the tail rotor. The signals were then high-pass filtered at $250 \mathrm{~Hz}$ in order to remove the lower harmonic loading and thickness noise from the signal. The averaging technique is described in greater detail in Reference 21. Agreement between the measured and modeled amplitudes and pulse shapes is very good.

Figure 6 also compares the BVI noise predicted by FRAME for the same steady-state descent at 60 KIAS and $-7.5^{\circ}$ flight-path angle but when the helicopter was $500 \mathrm{ft}$ ahead of the microphone array and 325 feet above ground level. Once again, the measured data are captured at observer positions towards the advancing, center, and retreating sides of the main rotor. However, because these microphone locations are closer to the helicopter and the angle between the rotor plane and the observers greater, the levels are noticeably larger. Once again, agreement is quite good, especially for the strong BVI observed by the advancing side microphone. It is clear from this quick check of the noise radiated under steady-state conditions that BVI is accurately modeled by the FRAME approach.

\section{Transient Maneuver FRAME Modeling}

In order to extend the FRAME approach to maneuvering flight conditions, the existing steady-flight BVI model must be replaced with a model that can account for timevarying changes in the rotor operating condition. The simplest method for doing this is to build a sequence of constant acceleration steady-state models that represent the helicopter as it transitions from one helicopter state to another. This is the essence of the Q-SAM method that has been shown to be valid for constant acceleration maneuvers. For a helicopter undergoing constant acceleration, the inflow through the rotor reaches an equivalent steady-state condition, which results in an equivalent steady-state fixed wake geometry. This equivalent geometry is used to find the equivalent steady-state operating condition. The radiated noise is then propagated to observers for the equivalent steady-state operation. Noise from these equivalent conditions make up constant acceleration segments that allow the helicopter to transition from one steady-state operating condition to another. The quasi-static method has only been recommended for slow-to-moderate maneuvers where the geometry of the wake does not change significantly during the maneuver.

In reality, the maneuvering helicopter is constantly undergoing accelerations along the flight-path. Therefore, the inflow through the rotor is also continually changing and never reaches the equivalent steady-state geometry of a constant acceleration maneuver. For slowly varying changes in acceleration, neglecting the dynamic motion of the wake would be expected to produced only small differences in the radiated noise.

A new method for approximating the effects of acceleration along the flight-path is suggested in this paper, 


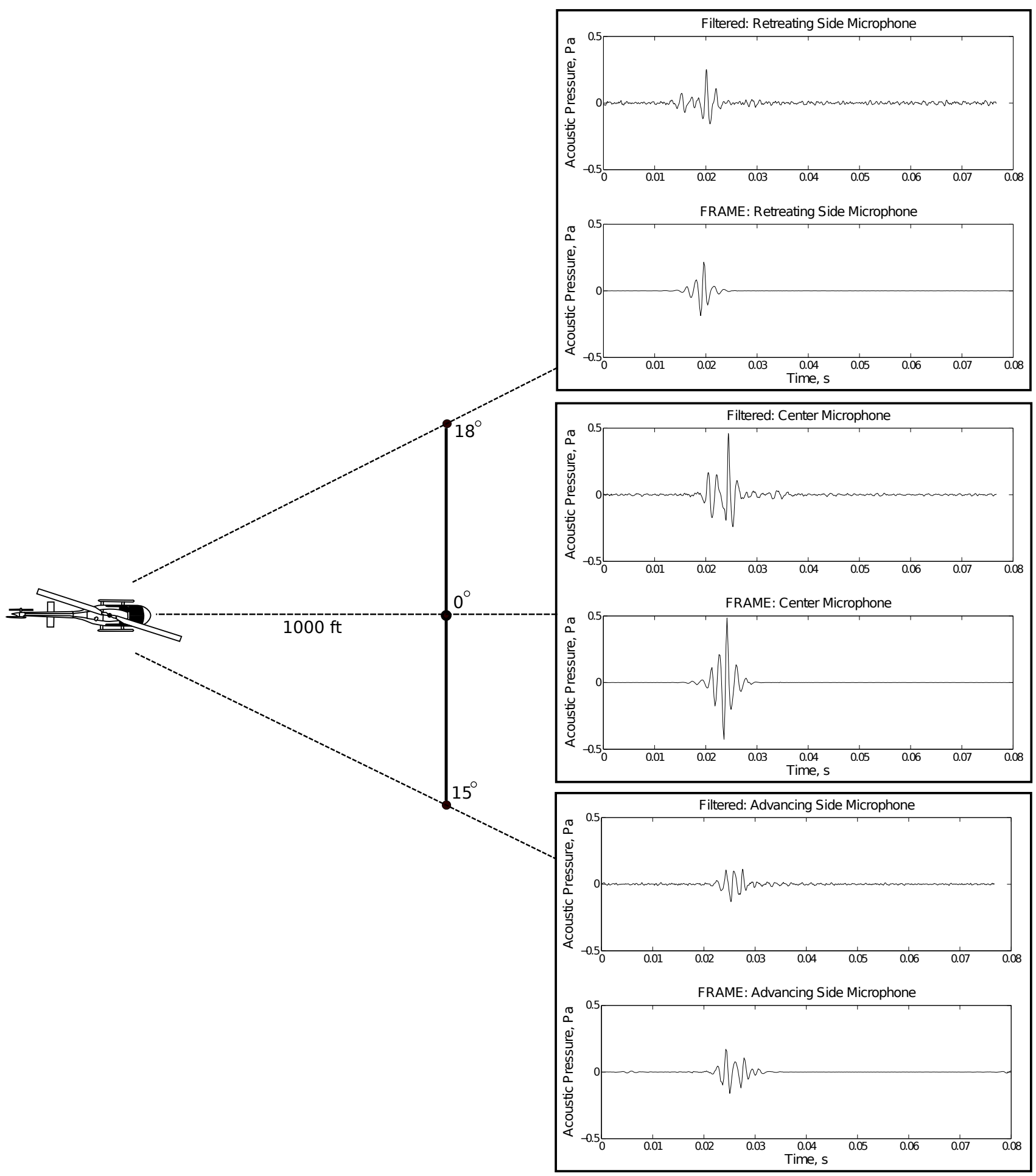

Fig. 5. Comparison of measured and FRAME pressure time histories emitted by the helicopter $1,000 \mathrm{ft}$ in front of the microphone array during steady $60 \mathrm{KIAS},-7.5^{\circ}$ flight-path angle descending flight.

FRAME-Dynamic (FRAME-D), which is a conceptual extension to the Q-SAM approach. As in Q-SAM, changes in acceleration require that the governing force balance equations hold at every time step during the maneuver. The effective controlling parameters of the problem are the main rotor tip-path-plane angle and thrust. Rotor inflow is determined at these changing conditions as in the Q-SAM approach. However, the vertical positions of the shed vortices with respect to the tip path plane of the rotor are now found by integrating the sum of the freestream velocity and the vertical induced inflow through the rotor disk. This inflow is continually changing as the force balance of the rotor determines the tip-path-plane angle and thrust magnitude of the maneuver. If steady-state conditions occur, the vertical positions of the wake return to their steady-state values. In addition, significant changes in the thrust will lead to corre- 


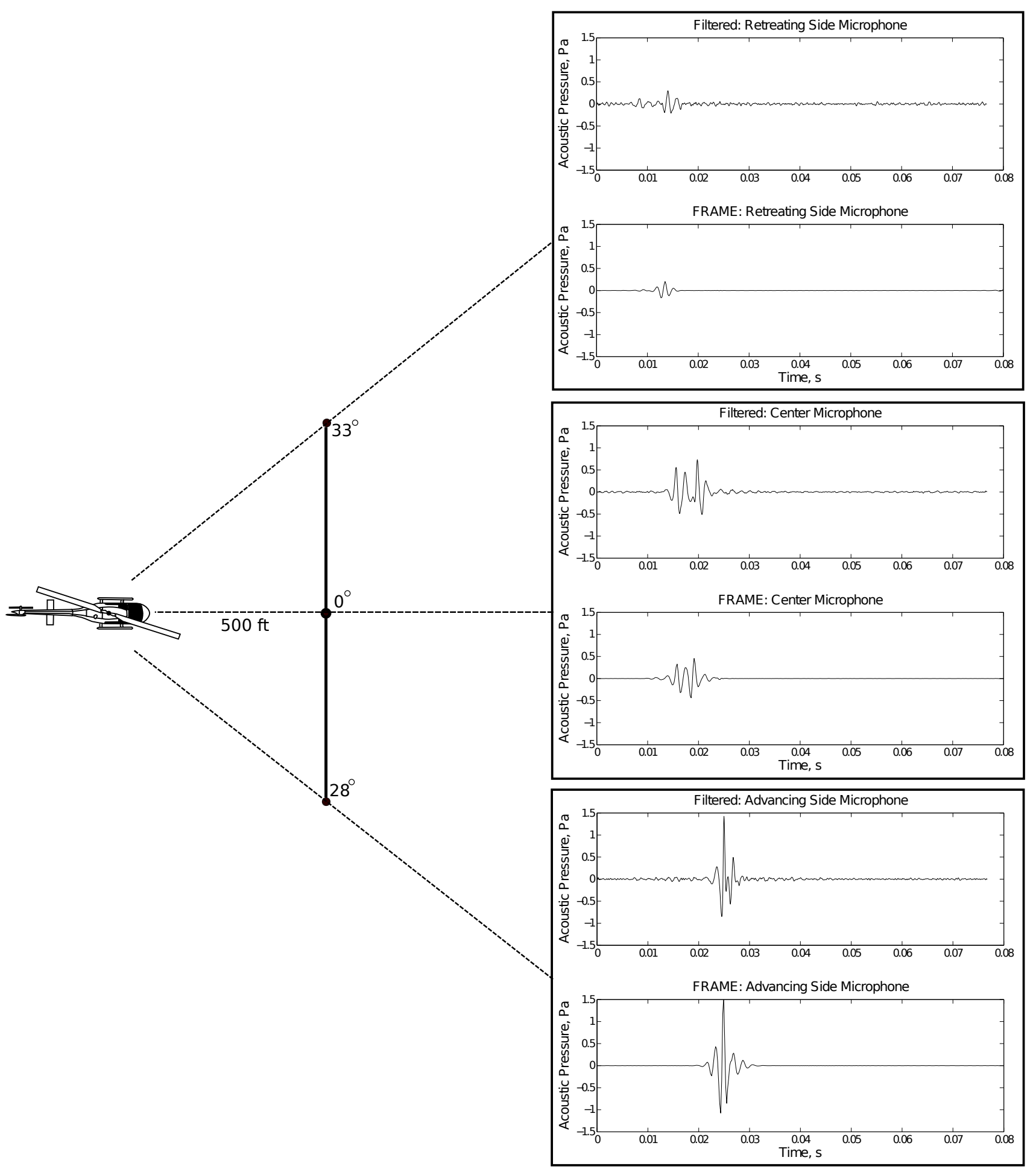

Fig. 6. Comparison of measured and FRAME pressure time histories emitted by the helicopter $500 \mathrm{ft}$ in front of the microphone array during steady $60 \mathrm{KIAS},-7.5^{\circ}$ flight-path angle descending flight.

sponding changes in the circulation strength of the tip vorticies and the amplitude of BVI noise.

The particular steady-state wake model that is altered in FRAME-D to account for transient dynamic maneuvers is a generalization of the prescribed wake concept of Beddoes. The vertical positions of tip vortex locations are described using an ODE that describes their convection from the ro- tor tip-path-plane due to the time-varying free-stream velocity of the rotor, rotation of the tip-path-plane through the medium, and induced inflow velocity. This generalization of the wake model captures the the change in the curvature of the wake due to rapid pitching or rolling of the tip-pathplane. In addition, the in-plane geometry of the shed tip vortices reflects the continuos variation in the advance ratio caused by a rapid acceleration or deceleration. The new 
dynamic wake model supplies input to the indicial aerodynamics method and acoustic solver as in the steady-flight version of FRAME. No additional modeling parameters are required to construct the model for transient flight, enabling existing steady-flight wind tunnel and flight test data to be used to construct FRAME-D models applicable to maneuvering flight conditions. A complete description of the mathematical model used by FRAME-D is supplied in the Appendix. Figure 7 is a flowchart describing how this timevarying model is utilized in conjunction with FRAME-D in this paper, with references to the relevant equations found in the Appendix

\section{Transient Maneuvering Flight FRAME-D Noise Predictions Versus Measurements}

The results of using the FRAME-D model to estimate the BVI noise generated by the Bell 206B for three maneuvers flown during the 2007 flight test program are compared with measurements of the radiated noise. The maneuvers considered in this paper include a fast cyclic pitch up, a slow cyclic pitch up and a fast cyclic roll towards the advancing side of the rotor. The inputs to the model were the inertial measurements of the position and orientation of the helicopter as well as the direct measurement of the longitudinal flapping of the rotor tip-path-plane. Winds were assumed to be zero in the model; during the test, winds were measured on the ground between 1 and $1.5 \mathrm{kts}$ in a crosswind direction. The enhanced FRAME-D model can be executed faster than real-time using one typical $2.6 \mathrm{GHz}$ desktop processor core for each observer at the resolution used to generate the results shown in this paper.

\section{Fast Longitudinal Cyclic Pitch Up}

The upper plots for each of the advancing side, center, and retreating side microphones shown in Figure 8 display the pressure time-histories measured during the fast pitch up maneuver. The maneuver was performed from level flight at an initial altitude of 150 feet starting from a distance of 1,700 feet from the microphone array and continued until the helicopter was 1,000 feet away from the array. Performing the maneuver causes a significant increase in the radiated noise. On the center microphone, two peaks in the BVI noise levels are observed; the first as the wake moves up through the rotor shortly after the initial pull up and a second after the wake passes back down through the rotor once the pilot begins to exit the pitch-up condition. The behavior on the retreating side is similar, shown on the uppermost plot, although with a greatly reduced second peak. On the advancing side, the peak noise level is found at a time between the two peaks observed by the center microphone.

The FRAME-D predictions are shown below the measured pressure-time histories in the same figure. The model captures the behavior of the BVI noise induced by the maneuver in both time and amplitude. As the maneuver progresses, the FRAME-D model captures the increase, then decrease, and then increase in peak amplitude on the center microphone that is shown in the data. The large increases in BVI noise of this $1.8 \mathrm{~g}$ maneuver are comparable to the levels obtained at the same non-dimensional distances during maximum BVI levels measured in steady descending flight.

The "double-dip" nature of the BVI can be rationalized by looking at the closest miss-distances between the vortex positions and the blades. Figure 9 shows a time-history plot of the vertical miss-distances between the shed tip vortices and the rotor blades in the advancing side rear quadrant of the rotor. The interactions caused by the vortex shed by one blade are red and those of the other blade black. There are four rows of miss distances; the upper most row are due to vortices with a wake age of about $180^{\circ}$ and each subsequent row is due to vortices about $180^{\circ}$ older than the last. In this figure, a negative miss-distance indicates a vortex below the rotor tip-path-plane and a positive miss-distance a vortex above it. As the helicopter pitches up, the tip-pathplane angle of attack increases, decreasing the mean inflow through the rotor. The vortices are seen to convect well above the plane of the rotor during the pitch up. This results in two times when the miss-distance approaches zero, at about zero and two seconds from the beginning of the maneuver. The peak levels of the noise radiated towards the center microphone occur when the miss distances are near zero-first when the vortices pass up through the rotor disk, and then again when they pass back under the disk. When the time delay of approximately one second due to propagation is accounted for, this corresponds to the observed timing of the BVI peaks in the pressure time-histories shown in Figure 8 Interestingly, the miss-distances of vortices at different ages tend to converge to zero at about the same time- this is thought to be because the higher pitch rate of the fast maneuver tends to cancel some of the wake curvature caused by the longitudinal linear induced inflow distribution. This may result in more BVI being activated simultaneously than in steady descending flight with the same mean inflow, especially for multi-bladed rotor systems where there are more trailed tip vortices.

Figure 10 shows an expanded time-history of the BVI pulses for approximately one revolution at a time near the maximum observed BVI amplitude for each observer. The BVI pulse shapes are seen to be similar for both the model predictions and measured data. Almost all of the important BVI events have been captured during this transient cyclic pull-up maneuver, although close examination shows that the model consistently overestimates the widths of the negative peaks. Note that the measured data contain noise generated by sources other than BVI, such as the tail rotor, whereas the estimated noise only includes the contribution of loading noise due to BVI. In addition, the rotor azimuth in the model is not synchronized with the measured data 


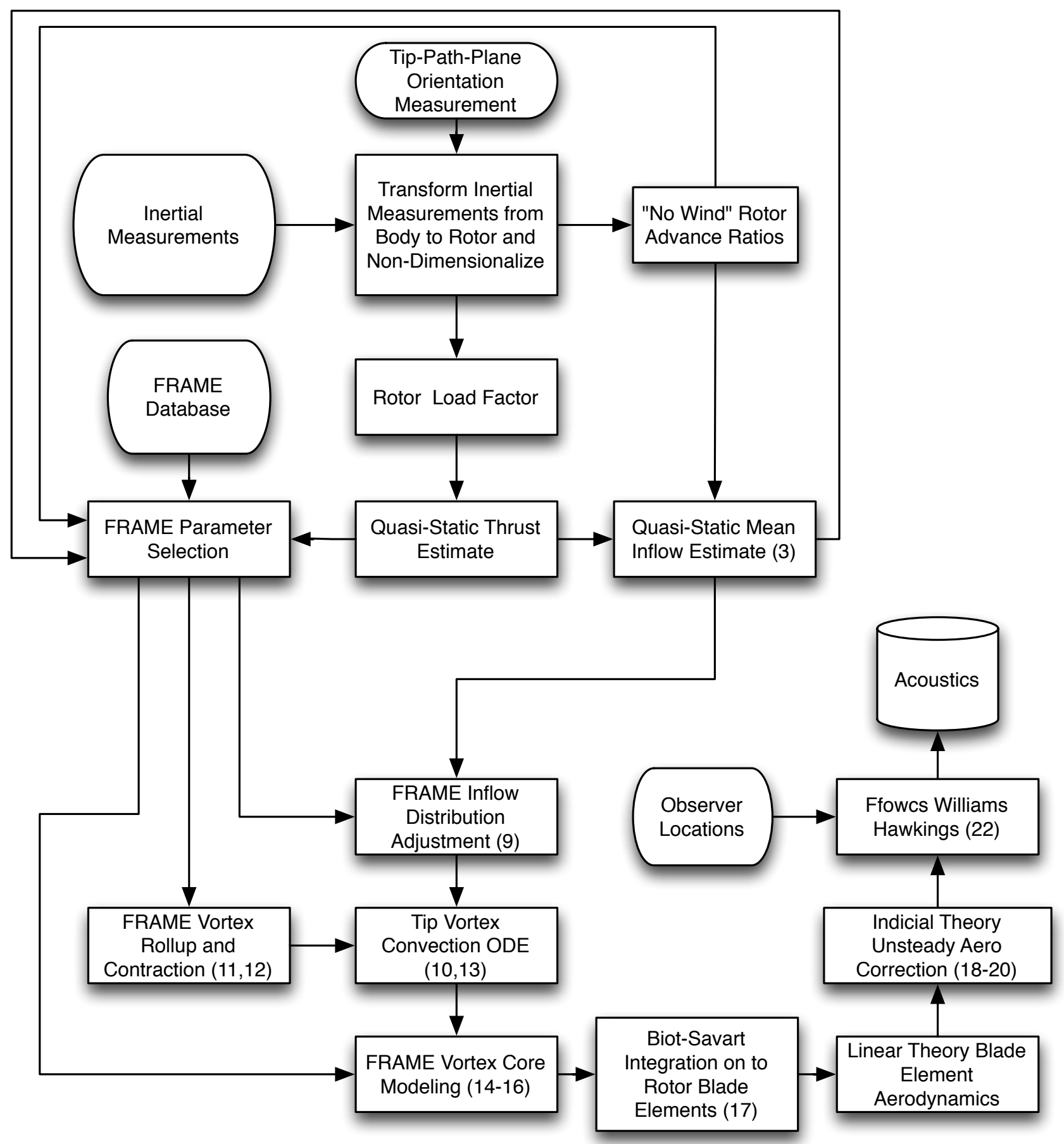

Fig. 7. A flowchart of the FRAME-D maneuver noise model, as used in this paper. Equation numbers are noted in blocks where applicable, and refer to the mathematical model detailed in the Appendix.

and as such the pulses do not align in phase.

Once validated with experiment, the FRAME-D model can also be used to estimate noise levels over a wide area. Figure 11 shows a sequence of ground noise contour plots, each plotted in OASPL calculated over a time period containing three revolutions of BVI noise estimated by the model. Figure 11a shows the noise levels just prior to onset of the maneuver from a steady level flight condition heading towards the southeast. Figure $11 \mathrm{p}$ shows the initial increase in BVI noise radiated ahead of the helicopter, setting the initial peaks on the center and retreating side microphones. Later, shown in Figure 11, the directivity has shifted to- wards the advancing side as the parallel BVI is activated, which sets the peak on the advancing side channel. As the helicopter ends the maneuver, the wake returns through the rotor, activating an oblique BVI, shown in Figure 11 d. The directivity of the noise shifts back towards the front of the helicopter, setting the second peak on the advancing and retreating side microphones.

\section{Slow Longitudinal Cyclic Pitch Up}

Figure 12 compares the pressure time histories measured by the advancing side, center, and retreating side ground 


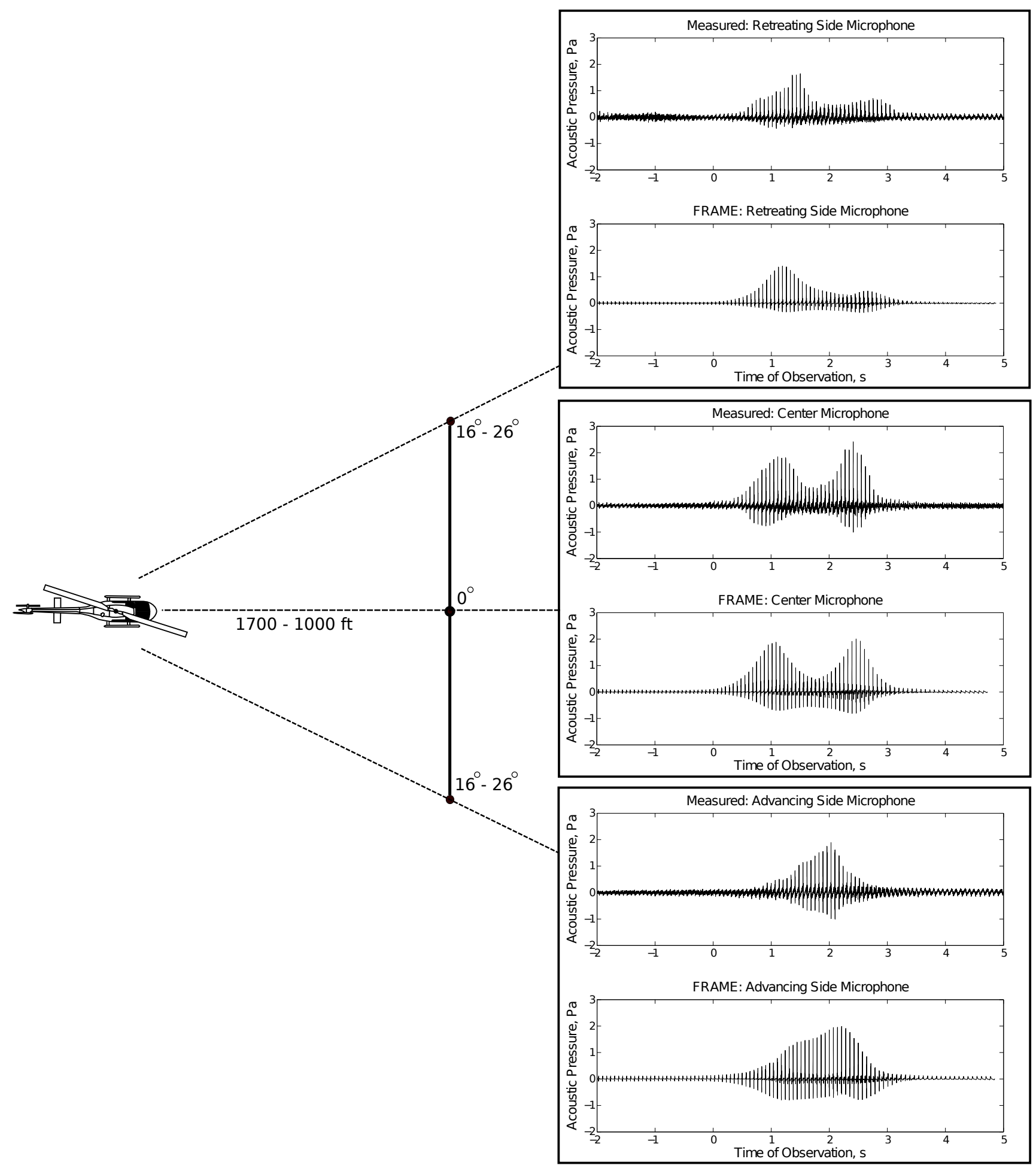

Fig. 8. Comparison of measured and FRAME predicted pressure time histories at microphone array locations over the duration of the fast pitch up maneuver.

based microphones to the pressure time histories estimated using the FRAME-D model for the Bell 206B during the slow pitch up maneuver. Once again, the helicopter begins the run in a steady level flight condition at an altitude of 150 feet above ground level. In general, the amplitudes of the BVI pulses are in good agreement between the measurement and the modeled pressure time histories, although the model overestimates the magnitude of BVI radiated towards the advancing side. The model does not capture some of the unsteadiness of the actual maneuver which results in less fluctuation in the BVI pulse amplitudes from one blade passage to the next. This may be caused in part by the low sampling rate of the position tracking data used to derive estimates of the helicopter's flight state, $1 \mathrm{~Hz}$, which is too 


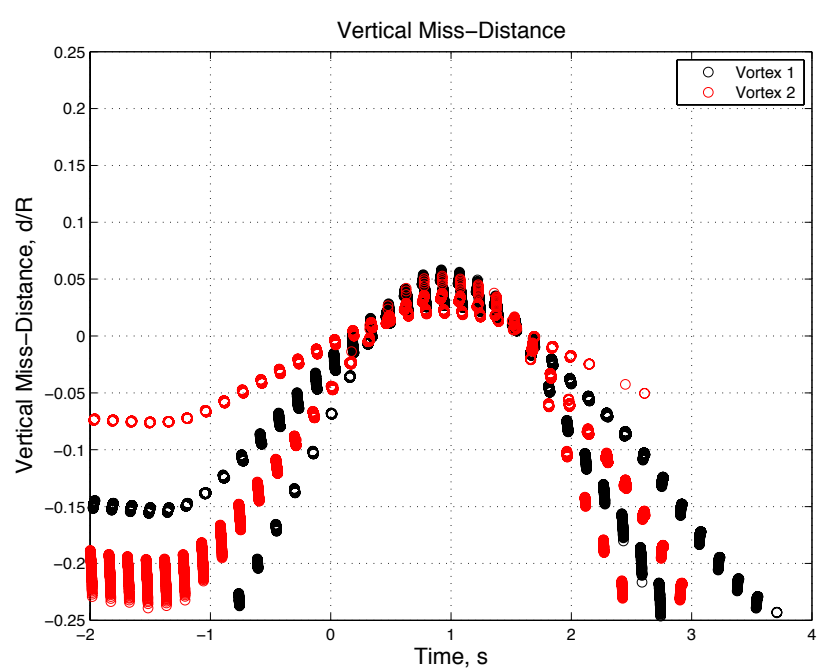

Fig. 9. Vertical miss-distance time history during fast pitch-up maneuver.

coarse to capture the pulse-to-pulse fluctuations. The model accurately captures the timing of the BVI noise generated by the maneuver, with peak noise seen first on the center and retreating side observers and followed almost two seconds later on the advancing side.

Figure 13 shows a time-history plot of the vertical missdistances between the shed tip vortices and the rotor blades in the advancing side rear quadrant of the rotor. In contrast to the fast pitch up, the vortices only just reach the rotor tip path plane, and the interactions with the younger vortices tend to have smaller miss-distances than those of the older vortices throughout the entire maneuver-this explains the lower noise levels on the advancing side and the presence of a single peak of BVI noise of a longer duration observed on the center and retreating side microphones.

\section{Fast Lateral Cyclic Advancing Side Roll}

Figure 14 compares the pressure time histories measured by the advancing side, center, and retreating side ground based microphones to the pressure time histories estimated using the FRAME-D model for the Bell 206B during a fast cyclic roll towards the advancing side initiated from level flight at a height of 150 feet above the ground. In general, the amplitudes of the BVI pulses are in good agreement between the measurement and the modeled pressure time histories, although the model overestimates the magnitude of BVI radiated towards the advancing side. This may be caused in part by a lack of lateral tip-path-plane information and the slow rate of the inertial measurement data used as an input to the model.

Plotted in Figure 15 is the time-history of vertical missdistances between the vortices and blades in the advancing side rear quadrant of the rotor during the fast advancing side roll. The miss-distance time-history is similar to that of the slow cyclic pitch up maneuver, previously shown in Figure
13 During the maneuver, the vortices just reach the tippath-plane in the advancing side rear quadrant, leading to a single BVI noise peak radiated towards the center microphone as seen in the ground noise measurements. The roll maneuver is somewhat more abrupt than the slow pitch-up, resulting in a BVI noise peak of shorter duration.

\section{Conclusions}

A new semi-empirical analytical model was introduced in this paper that describes the generation and radiation of BVI noise for a single main rotor helicopter executing two different kinds of transient maneuver. The model is an extension of a steady-state parameter identification modeling method that is based upon the basic physics of BVI noise radiation. The model includes the effect of accelerated flight in the resulting force balance equations, but also includes the integrated effects of the tip vortex positions to determine the miss distance between the tip vortices with respect to the blades.

The change in BVI caused by maneuvers appears to be primarily caused by the change in miss-distance between the rotor blades and tip vortices and also by the changing thrust of the main rotor. For the rapid cyclic pull-up of the Bell 206B helicopter, the model captures both the qualitative and quantitative features of the radiated noise. Peak amplitudes and pulse shapes during the transient maneuver are well captured at three microphone locations.

The slower cyclic maneuver results also capture the major trends measured in the test program-but not as well as the fast maneuver case, due to the variability of the measured BVI noise. The event is less discernible in the measurements because strong BVI are not observed. The BVI produced during a pure cyclic roll transient maneuver are also predicted fairly well. However, the predicted levels are too high towards the advancing side, suggesting that more improvements of the modeling of turning flight are probably required.

The general concept of modeling of transient maneuvering flight based upon measured steady data has been shown to be viable. This simplified method developed is based upon the physics of the problem and is both computationally fast and accurate. It is a good candidate for use in realtime displays and for developing noise exposure forecasts of a maneuvering helicopter.

\section{Appendix: Mathematical Description of the FRAME-D BVI Noise Model}

The FRAME-D BVI noise model is an extension of the steady-state BVI noise model used in a previous version of FRAME, which was based on the Beddoes' prescribed wake model. In the Beddoes' wake model, the position of a vortex element in the rotor wake is a function of the steadystate flight condition. The new wake model in FRAME$\mathrm{D}$ extends the Beddoes' concept to maneuvering flight by 


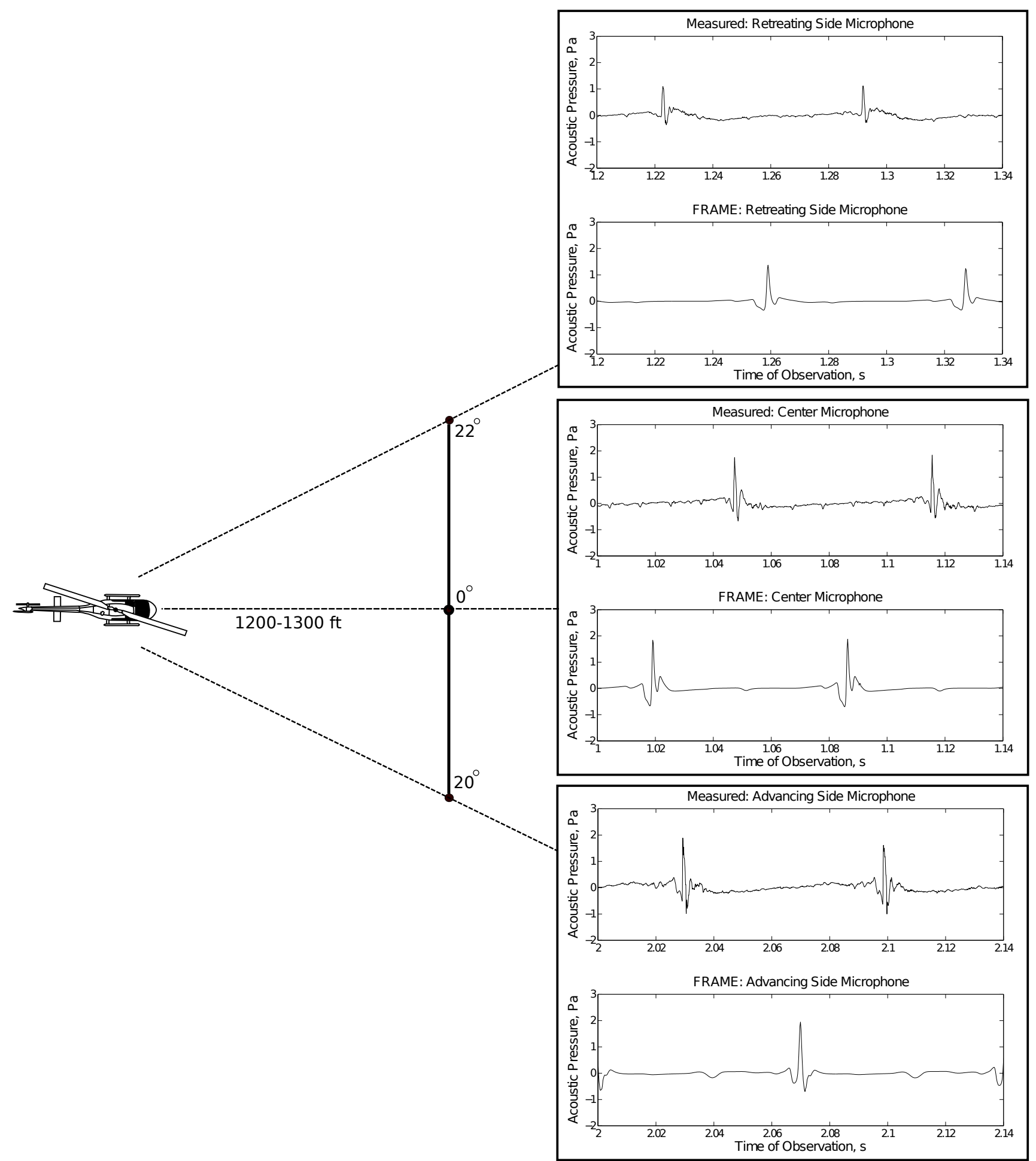

Fig. 10. Comparison of measured and FRAME predicted BVI pulses at microphone array locations near the peak observed levels during the fast pitch up maneuver.

determining the position of a vortex element through the time integration of the pitching and rolling motions of the tip-path-plane, the free stream velocity, and the vertical induced velocity over the wake age of the vortex element. For a steady-state flight condition, this new model is equivalent to the modified Beddoes model previously used in FRAME.

As in the Beddoes' wake, an induced inflow distribution is prescribed over the rotor a priori. The trailed tip vortices are then assumed to convect with the superposition of the induced velocity and the motion of the rotor with respect to the medium. Unlike Beddoes' model, the flight condition is allowed to vary over time and the rotor tip-path-plane to rotate in space. The vertical motion of the vortex elements in tip-path-plane coordinates ( $\mathrm{x}$-aft, $\mathrm{y}$-advancing side, $\mathrm{z}$-up) 


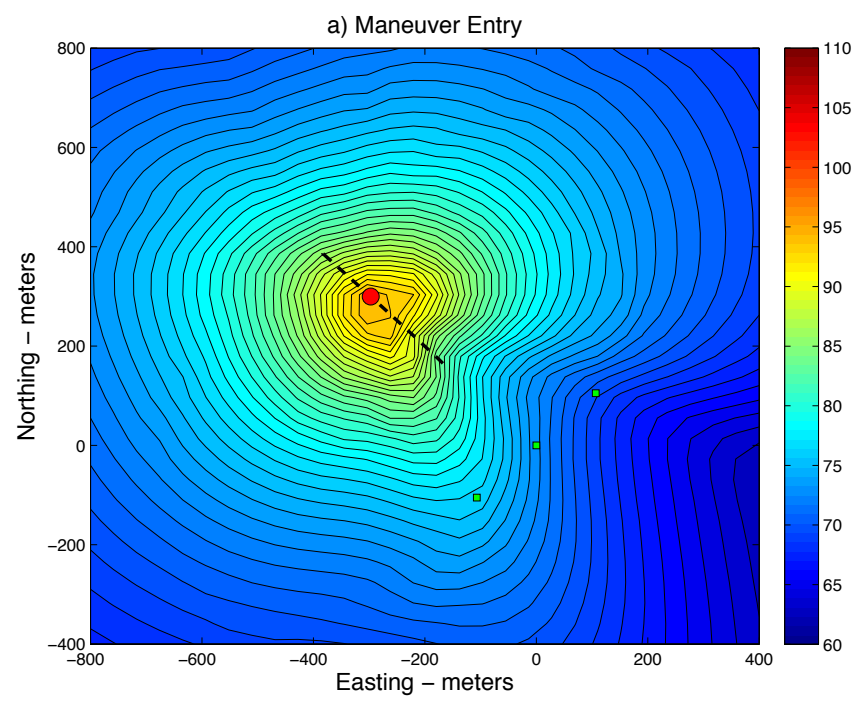

c) Peak on Advancing Side Microphone

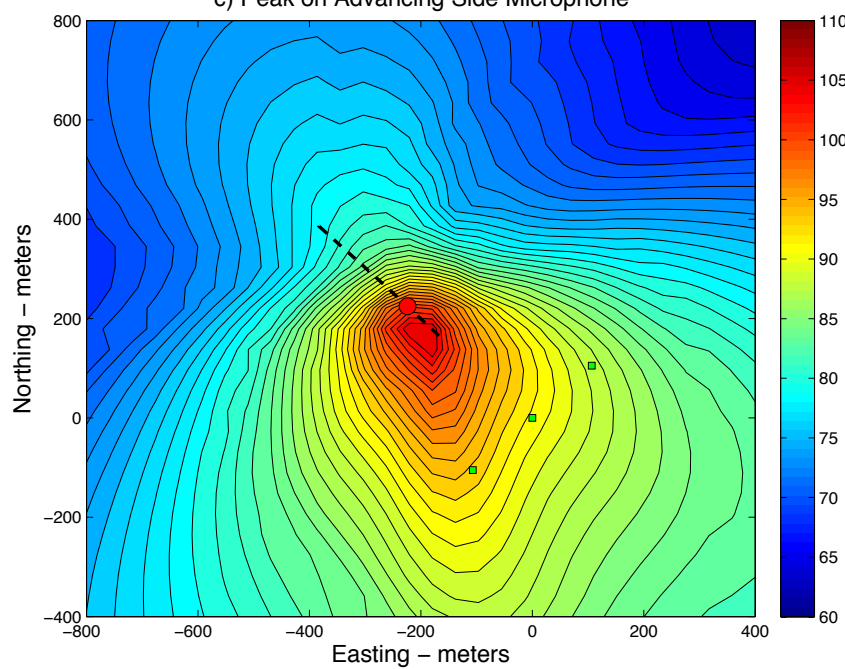

b) Beginning of First Peak on Center Microphone

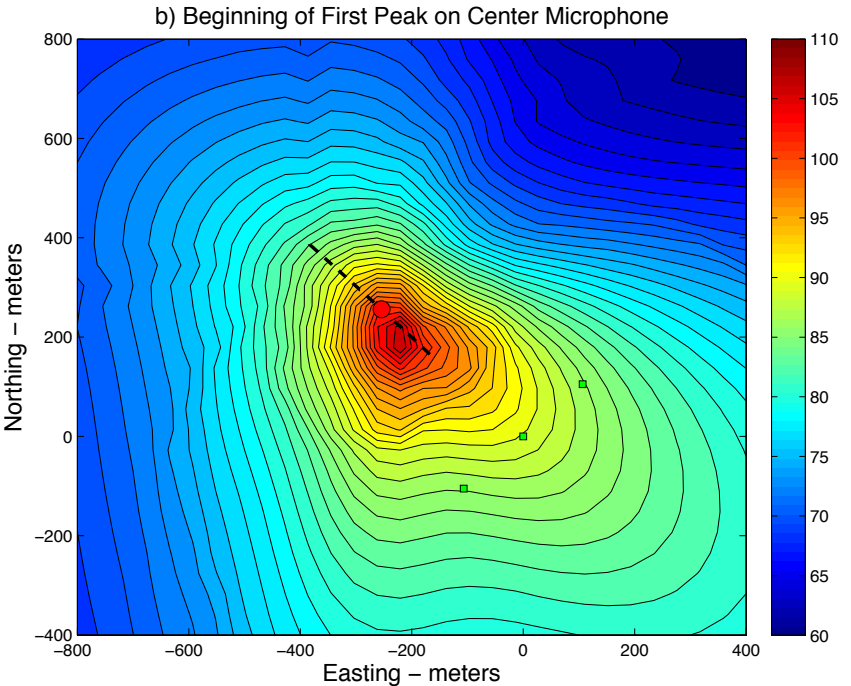

d) Second Peak on Center Microphone

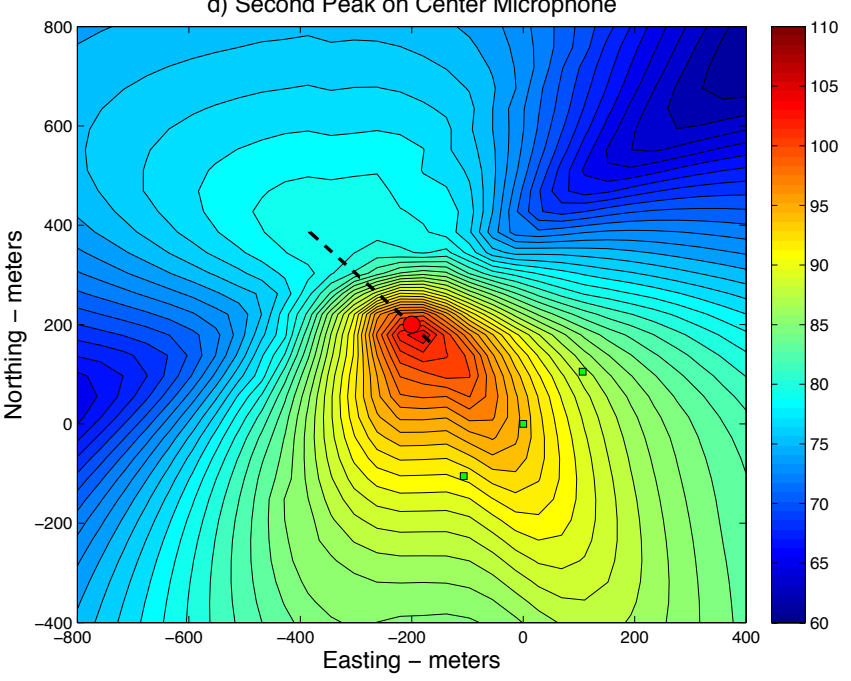

Fig. 11. Sequence of BVI ground noise contour plots (OASPL) for the fast pitch up. Black dashed line is the helicopter trajectory, the red circle is current helicopter position, and the green squares are microphone locations.

is therefore described by the following differential equation:

$$
\frac{\partial z_{v}}{\partial \psi}=\left(\vec{\omega}(\psi) \times \vec{x}_{v}(\psi)\right) \cdot \hat{k}+\mu_{z}(\psi)-\lambda_{i}\left(\vec{x}_{v}(\psi), \psi\right)
$$

The Beddoes induced inflow distribution across the rotor disk is the superposition of the mean induced inflow, the longitudinal linear Glauert inflow distribution (Ref.22), and Beddoes' lateral cubic inflow distribution representing the cumulative upwash induced by the rolled-up trailed tip vortices.

$\lambda_{i}\left(\vec{x}_{v}(\psi), \psi\right)=\lambda_{0}(\psi)+\lambda_{1 c}(\psi) x_{v}(\psi)-\lambda_{1 c}(\psi)\left|y_{v}^{3}(\psi)\right|$

where the mean induced inflow is determined from momentum theory and Glauert's linear longitudinal inflow coefficient is taken to be the wake skew angle:

$$
\lambda_{0}=\frac{C_{T}}{2 \sqrt{\left(\lambda_{0}-\mu_{z}\right)^{2}+\mu_{x}^{2}+\mu_{y}^{2}}}
$$

$$
\lambda_{1 c}=\chi=\arctan \frac{\mu_{x}}{\lambda_{0}-\mu_{z}}
$$

For a steady flight condition with no pitch or roll rate, and where the tip-vortices are released from the blade tips (i.e. $r=1$ ), this model is identical to Beddoes' algebraic model for the vertical position of vortices underneath the rotor disk. When integrated for a steady-flight condition, the model yields the following algebraic expression:

$$
\begin{gathered}
z_{v}(\phi)=\mu_{z} \phi-\int_{\psi_{b}}^{\psi_{v}} \lambda_{i} d \psi \\
=\phi\left[\mu_{z}-\lambda_{0}-\lambda_{1 c}\left(\cos \psi_{v}+\frac{\mu_{x}}{2} \phi-\left|\sin \psi_{v}\right|^{3}\right)\right]
\end{gathered}
$$

Further experimental studies of rotor wakes have suggested revisions to the Beddoes model. For example, van der Wall [17] found that a lateral linear distribution of induced inflow superimposed on the Beddoes distribution improved agreement of the model with the HART PIV mea- 


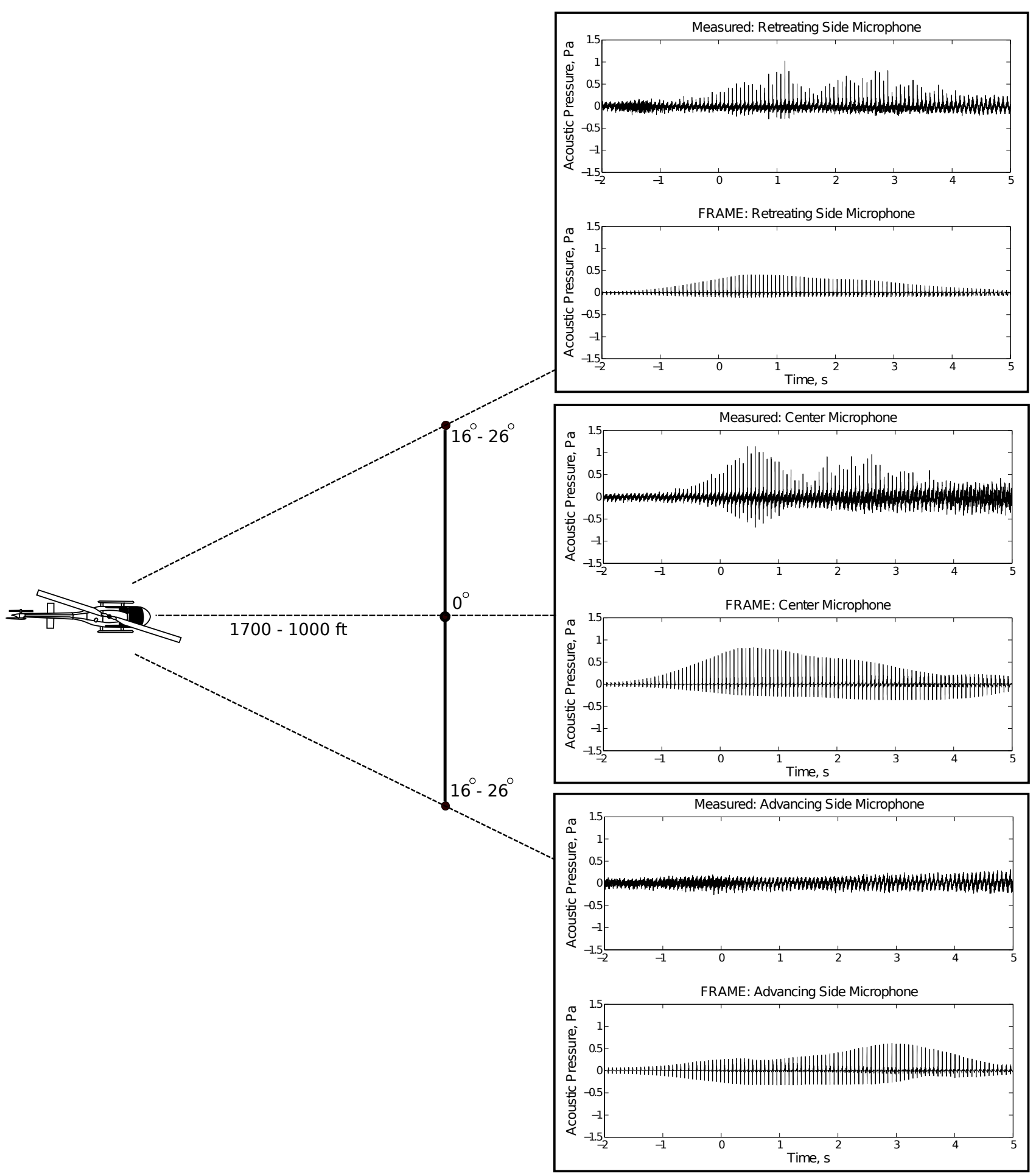

Fig. 12. Comparison of measured and FRAME predicted pressure time histories at microphone array locations over the duration of the slow pitch up maneuver.

surements of the rotor wake.

$$
\begin{aligned}
\lambda_{i}\left(\vec{x}_{v}(\psi), \psi\right)= & \lambda_{0}(\psi)+\lambda_{1 c}(\psi) x_{v}(\psi) \\
& -\lambda_{1 c}(\psi)\left|y_{v}^{3}(\psi)\right|+\lambda_{1 s}(\psi) y_{v}(\psi)
\end{aligned}
$$

where the lateral inflow coefficient is that suggested by Drees' inflow model (Ref. 23) and the longitudinal coef- ficient reduced to half the wake skew.

$$
\begin{gathered}
\lambda_{1 c}=\frac{\chi}{2} \\
\lambda_{1 s}=-2 \mu_{x}
\end{gathered}
$$

In practice, the best inflow distribution coefficients for a specific vehicle configuration are not known a priori. A 


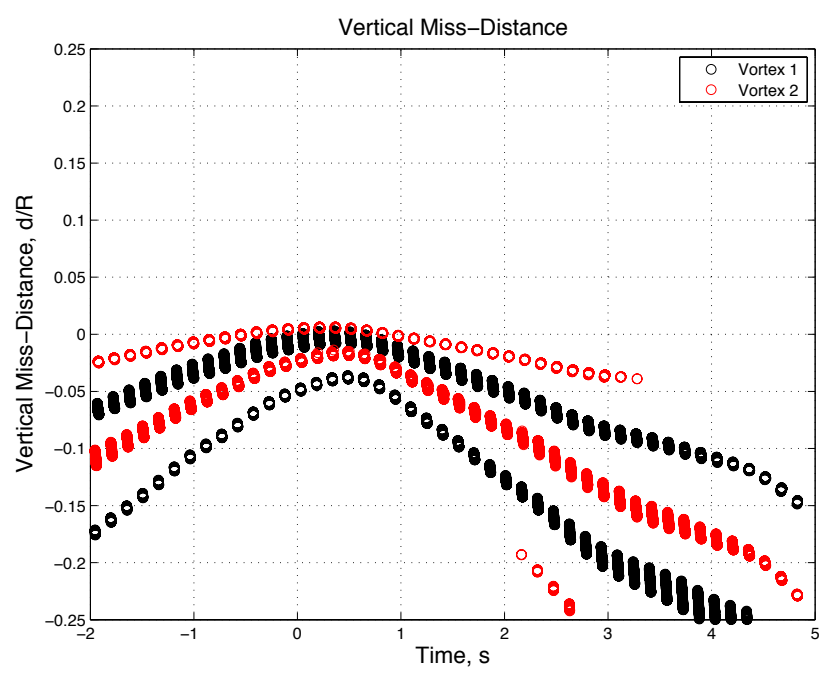

Fig. 13. Vertical miss-distance time history during slow pitch-up maneuver.

range of potential inflow distributions may be described more generally as the following.

$$
\begin{aligned}
\lambda_{i}\left(\vec{x}_{v}(\psi), \psi\right)= & A \lambda_{0}(\psi)+B \lambda_{1 c}(\psi)\left[x_{v}(\psi)-\left|y_{v}^{3}(\psi)\right|\right] \\
& +C \lambda_{1 s}(\psi) y_{v}(\psi)
\end{aligned}
$$

where $A, B$, and $C$ are empirical parameters which control the respective mean, longitudinal, and lateral components of the induced inflow distribution over the rotor disk.

In addition to the vertical position of the trailed tip vortices with respect to the tip-path-plane, the longitudinal and lateral motion of the wake effects BVI. The epicyclical geometry of the wake sets the interaction angles between the blades and the vortices, which determines how the acoustic disturbances accumulate in the far field and the resulting directivity and magnitude of BVI noise.

While Beddoes' original model assumed that the blade tip vortices were trailed from the tip of the blade, in practice the tip-vortices roll-up somewhat inboard of the blade tips. Therefore, the initial position of a vortex element at the time of release can be described as follows:

$$
\vec{x}_{v}\left(\psi_{0}\right)=\left\{\begin{array}{l}
x_{v}\left(\psi_{0}\right) \\
y_{v}\left(\psi_{0}\right) \\
z_{v}\left(\psi_{0}\right)
\end{array}\right\}=\left\{\begin{array}{c}
r_{r} \cos \psi_{0} \\
r_{r} \sin \psi_{0} \\
0
\end{array}\right\}
$$

As the rotor wake convects downstream, it also contracts. This can be accounted for by applying an empirical model of the wake contraction. Landgrebe and Egolf identified the following algebraic model for the wake contraction in forward flight. (Refs. 24, 25)

$$
\begin{gathered}
r_{v}=r_{r}\left(D+(1-D) e^{-\lambda_{1} \phi}\right) \\
\lambda_{1}=0.145+27 C_{T}
\end{gathered}
$$

where $D$ is an empirical parameter controlling the rate of wake contraction.

Altogether, the differential equation describing the convection of a vortex element relative to the rotor hub due to rotation, translation, and induced velocities is as follows:

$$
\begin{aligned}
& \frac{\partial \vec{x}_{v}}{\partial \psi}=\vec{\omega}(\psi) \times \vec{x}_{v}(\psi)+\vec{\mu}(\psi) \\
& -\left\{\begin{array}{c}
(1-D) \lambda_{1} e^{-\lambda_{1}\left(\psi_{b}-\psi_{v}\right)} x_{0} \\
(1-D) \lambda_{1} e^{-\lambda_{1}\left(\psi_{b}-\psi_{v}\right)} y_{0} \\
\lambda_{i}\left(\vec{x}_{v}(\psi), \psi\right)
\end{array}\right\}
\end{aligned}
$$

where $\lambda_{i}\left(\vec{x}_{v}(\psi), \psi\right)$ is detirmined using Equation 9

This ODE is solved for each vortex element from the time of release until it has passed behind the rotor disk and no longer contributes to BVI. For steady flight, the model reduces to the same algebraic model as was used previously for BVI noise estimation in FRAME. With the coefficients $A, B, C, D$ also set to unity, the Beddoes wake with Drees' lateral inflow is recovered.

In addition to the wake geometry, there are other mechanisms that control the strength of BVI noise. The initial vortex core size and growth rate control the diameter of viscous vortex core along the tip vortices, and thereby influence the impulsiveness of the BVI events and the resulting noise levels. The non-dimensional vortex core size is expressed in the model as:

$$
\bar{r}_{c}=\sqrt{\bar{r}_{0}^{2}+4 C_{v}\left(\psi_{b}-\psi_{v}\right)}
$$

The strength of the tip vortex directly influences the strength of the BVI, and varies azimuthally. This is expressed in the model using an additional three parameters:

$$
\bar{\gamma}_{v}\left(\psi_{v}\right)=\bar{\Gamma}_{0}\left(\gamma_{0}+\gamma_{1 s} \sin \psi_{v}+\gamma_{1 c} \cos \psi_{v}\right)
$$

where the nominal vortex strength $\bar{\Gamma}_{0}$ is determined by the ideal vortex strength resulting from a constant triangular load distribution along the blade span:

$$
\bar{\Gamma}_{0}=\frac{\Gamma_{0}}{\Omega R^{2}}=\frac{2 \pi C_{T}}{b}
$$

The velocity induced by the vortices onto the blades is then evaluated through integration of the viscous BiotSavart law in order to calculate the instantaneous lift coefficient at the blades due to BVI. The Vatistas (Ref. 26) model with $n=2$ is used, which can be described with the following relation for non-dimensional swirl velocity:

$$
\frac{v_{\theta}}{\Omega R}=\frac{\bar{\gamma}_{v} \bar{r}}{2 \pi\left(\bar{r}_{c}^{2 n}+\bar{r}^{2 n}\right)^{(1 / n)}}
$$

The induced velocities are then used to calculate the perturbation in the blade section angles of attack. The 2D incompressible Beddoes-Leishman indicial aerodynamics 


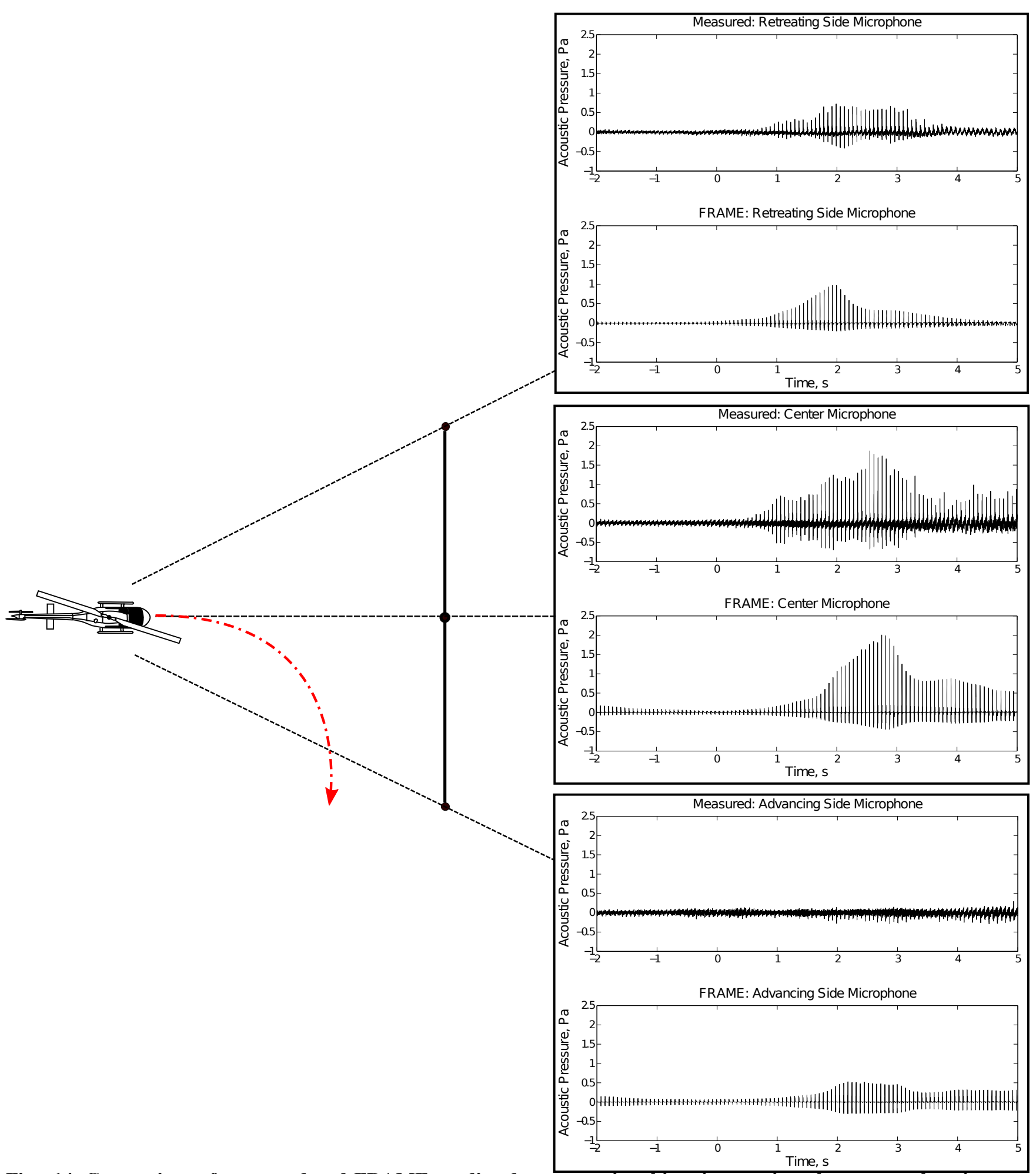

Fig. 14. Comparison of measured and FRAME predicted pressure time histories at microphone array locations over the duration of the fast advancing side roll maneuver. 


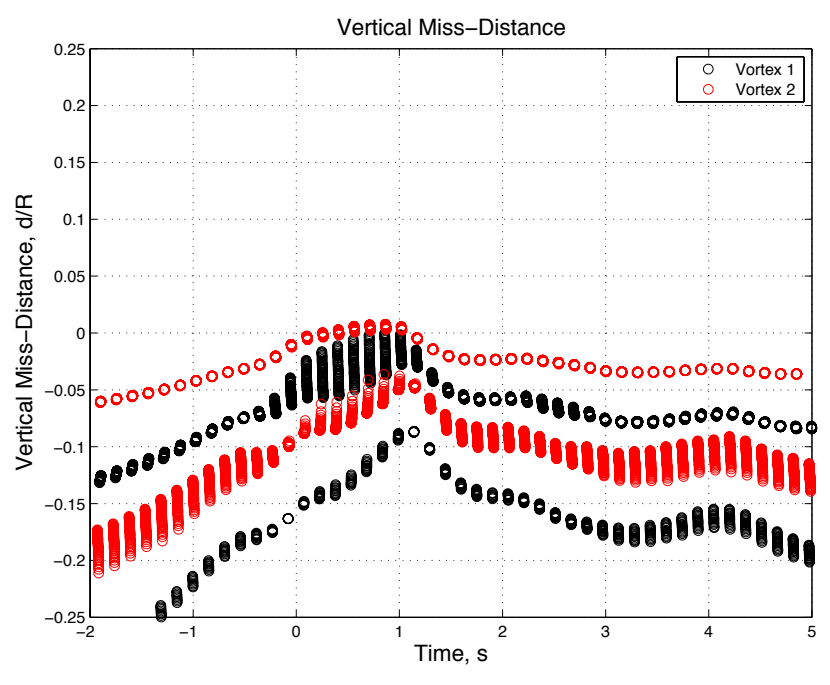

Fig. 15. Vertical miss-distance time history during fast advancing side roll maneuver.

method (Refs. 20, 27) is applied to the perturbation angles of attack to produce the airloads used to estimate BVI noise:

$$
\alpha_{e}(s)=\alpha(s)-X(s)-Y(s)
$$

where the indicial functions are expressed as:

$$
\begin{aligned}
& X(s)=X(s-\Delta s) e^{-b_{1} \Delta s}+A_{1} \Delta \alpha_{s} e^{-b_{1} \Delta s / 2} \\
& Y(s)=Y(s-\Delta s) e^{-b_{2} \Delta s}+A_{2} \Delta \alpha_{s} e^{-b_{2} \Delta s / 2}
\end{aligned}
$$

with respect to the reduced time, $s$, which is the distance traveled by the airfoil over a given time $t$ expressed in semichords, described by the following:

$$
s=\frac{2}{c} \int_{0}^{t} V d t
$$

The loading noise component of Formulation 1A of the Ffowcs Williams - Hawkings equation, (Ref. 28) below, is used to estimate BVI noise:

$$
\begin{aligned}
& 4 \pi p_{L}^{\prime}=\iint_{S} {\left[\left(\frac{1}{a_{0}} \frac{\dot{P}_{i j} \hat{n}_{j} \hat{r}_{j}}{r\left(1-M_{r}\right)^{2}}+\frac{P_{i j} \hat{n}_{j}\left(\hat{r}_{j}-\hat{M}_{j}\right)}{r^{2}\left(1-M_{r}\right)}\right.\right.} \\
&\left.\left.+\frac{1}{a_{0}} \frac{P_{i j} \hat{n}_{j} \hat{r}_{j}\left(r \dot{M}_{r}+a_{0}\left(M_{r}-M^{2}\right)\right.}{r^{2}\left(1-M_{r}\right)^{3}}\right) d S\right]_{\tau}
\end{aligned}
$$

Following Schmitz et al., (Ref. 16) this equation can be non-dimensionalized with respect to the rotor geometry and operating condition, yielding acoustic pressure nondimensionalized with respect to the ambient pressure:

$$
\begin{aligned}
4 \pi C_{p_{L}^{\prime}}=\iint_{\bar{S}} & {\left[M ^ { 2 } \left(\frac{M_{H} C_{P_{i j}} \hat{n}_{j} \hat{r}_{j}}{\bar{r}\left(1-M_{r}\right)^{2}}+\frac{C_{P_{i j}} \hat{n}_{j}\left(\hat{r}_{j}-\hat{M}_{j}\right)}{\bar{r}^{2}\left(1-M_{r}\right)^{2}}\right.\right.} \\
& \left.\left.+\frac{\left(\bar{r} M_{r} M_{H}+M_{r}-M^{2}\right) C_{P_{i j}} \hat{n}_{j} \hat{r}_{j}}{\bar{r}^{2}\left(1-M_{r}\right)^{3}}\right) d \bar{S}\right]_{\bar{\tau}}
\end{aligned}
$$

The parameters used by FRAME to fit the analytical model to the measured data for a particular operating condition are the unknown empirical terms $A, B, C, D, r_{r}, r 0, C_{v}$, $\gamma_{0}, \gamma_{1 s}$, and $\gamma_{1 c}$. FRAME is applied to BVI noise measurements for a range of steady operating conditions for isolated rotors in wind tunnels and full vehicles in flight test in order to develop a relation between the governing and dependent parameters applicable over a wide range of operating conditions. These parameters can then be employed in the FRAME-D model, varying with the quasi-static values of the governing parameters defining the rotor acoustic state, $\mu, \lambda_{0}, C_{T}$, and $M_{H}$.

\section{Acknowledgements}

The authors would like to thank Dr. Ben Wel-C Sim and Michael E. Watts for helpful discussions during the preparation of this paper.

\section{References}

${ }^{1}$ Lucas, M. J. and Marcolini, M. A., "Rotorcraft Noise Model," AHS Technical Specialists' Meeting for Rotorcraft Acoustics and Aerodynamics, October 1997.

${ }^{2}$ Conner, D. A. and Page, J. A., "A Tool for Low Noise Procedures Design and Community Noise Impact Assessment: The Rotorcraft Noise Model (RNM)," Heli Japan, 2002.

${ }^{3}$ Fleming, G. G. and Rickley, E. J., "Heliport Noise Model, HNM. Version 2.2 (User's Guide)," Technical report, Federal Aviation Administration, February 1994.

${ }^{4}$ Guntzer, F., Spiegel, P., and Lummer, M., "Genetic Optimizations of EC-135 Noise Abatement Flight Procedures using and Aeroacoustic Database," 35th European Rotorcraft Forum, September 2009.

${ }^{5}$ Gervais, M., Gareton, V., Dummel, A., and Heger, R., "Validation of EC130 and EC135 Environmental Impact Assessment using HELENA," American Helicopter Society 66th Annual Forum, May 2010.

${ }^{6}$ Browne, R. W., Munt, R. M., Simpson, C. R., and Williams, T., "Prediction of Helicopter Noise Contours for Land Use Planning," 10th AIAA/CEAS Aeroacoustics Conference, 2004.

${ }^{7}$ Gopalan, G., Quasi-Static Acoustic Mapping of Helicopter Blade-Vortex Interaction Noise, Ph.D. thesis, University of Maryland, 2004.

${ }^{8}$ Schmitz, F. H., Greenwood, E., Sickenberger, R. D., Gopalan, G., Sim, B. W.-C., Conner, D. A., Moralez, E., and Decker, W., "Measurement and Characterization of Helicopter Noise in Steady-State and Maneuvering Flight," American Helicopter Society 63rd Annual Forum, May 2007. 
${ }^{9}$ Page, J. A., Wilmer, C., and Plotkin, K. J., "Rotorcraft Noise Model Technical Reference and User Manual," Technical Report WR 08-04, Wyle, February 2008.

${ }^{10}$ Sickenberger, R. D., Gopalan, G., and Schmitz, F. H., "Helicopter Near-Horizon Harmonic Noise Radiation due to Cyclic Pitch Control," American Helicopter Society 67th Annual Forum, May 2011.

${ }^{11}$ Burley, C. L., Brooks, T. F., Charles, B. D., and McCluer, M., "TiltRotor Aeroacoustic Code (TRAC) Prediction Assessment And Initial Comparisons With Tram Test Data," 25th European Rotorcraft Forum, September 1999.

${ }^{12}$ Hennes, C. C., Chen, H.-N., Brentner, K. S., Ananthan, S., and Leishman, J. G., "Influence of Transient Flight Manuevers on Rotor Wake Dynamics and Noise Radiation," American Helicopter Society Specialists Conference on Aeromechanics, january 2004.

${ }^{13}$ Chen, H.-N., Brentner, K. S., Ananthan, S., and Leishman, J. G., "A Computational Study of Helicopter Rotor Wakes and Noise Generated During Transient Maneuvers," American Helicopter Society 61st Annual Forum, June 2005.

${ }^{14}$ Greenwood, E., Fundamental Rotorcraft Acoustic Modeling from Experiments (FRAME), Ph.D. thesis, University of Maryland, January 2011.

${ }^{15}$ Boxwell, D. A., Schmitz, F. H., Splettstoesser, W. R., and Schultz, K. J., "Model Helicopter Rotor High-Speed Impulsive Noise: Measured Acoustics and Blade Pressures," Technical Report TM 85850, NASA, September 1983.

${ }^{16}$ Schmitz, F. H., Boxwell, D. A., Léwy, S., and Dahan, C., "Model- to Full-Scale Comparisons of Helicopter BladeVortex Interaction Noise," Journal of the American Helicopter Society, Vol. 29, (2), 1984, pp. 16-25.

${ }^{17}$ Greenwood, E. and Schmitz, F. H., "A Parameter Identification Method for Helicopter Noise Source Identification and Physics-Based Semi-Empirical Modeling," American Helicopter Society 66th Annual Forum, May 2010.

18 van der Wall, B. G., "The Effect of HHC on the Vortex Convection in the Wake of a Helicopter Rotor," Aerospace Science and Technology, Vol. 4, (5), 2000, pp. 321-336.

${ }^{19}$ Beddoes, T. S., “A Wake Model for High Resolution Airloads," International Conference on Rotorcraft Basic Research, February 1985.

${ }^{20}$ Beddoes, T. S., "Practical Computation of Unsteady Lift," Vertica, Vol. 8, (1), 1984.

${ }^{21}$ Greenwood, E. and Schmitz, F. H., "Separation of Main and Tail Rotor noise Ground-Based Acoustic Measurements using Time-Domain De-Dopplerization," 35th European Rotorcraft Forum, September 2009.
${ }^{22}$ Glauert, H., "On the Horizontal Flight of a Helicopter," Technical Report RM 1157, ARC, 1928.

${ }^{23}$ Drees, J. M., "A Theory of Airflow through Rotors and its Application to Some Helicopter Problems," Journal of Helicopter Association of Great Britain, Vol. 3, (2), 1949.

${ }^{24}$ Landgrebe, A. J., "The Wake Geometry of a Hovering Helicopter Rotor and its Influence on Rotor Performance," Journal of the American Helicopter Society, Vol. 17, (4), 1972.

${ }^{25}$ Egolf, T. A. and Landgrebe, A. J., "Helicopter Rotor Wake Geometry and Its Influence in Forward Flight. Volume 1: Generalized Wake Geometry and Wake Effect on Rotor Airloads and Performance," Technical Report CR 3726, NASA, October 1983.

${ }^{26}$ Vatistas, G. H., Kozel, V., and Mih, W. C., "A Simpler Model for Concentrated Vortices," Experiments in Fluids, Vol. 11, (1), 1991, pp. 73-76.

${ }^{27}$ Leishman, J. G., Principles of Helicopter Aerodynamics, Cambridge University Press, New York, second edition, 2006.

${ }^{28}$ Farassat, F., "Derivation of Formulations 1 and 1A of Farassat," Technical Report TM-2007-214853, NASA, 2007. 